\title{
ANALYSIS OF A ROBUST FINITE ELEMENT APPROXIMATION FOR A PARABOLIC EQUATION WITH ROUGH BOUNDARY DATA
}

\author{
DONALD A. FRENCH AND J. THOMAS KING
}

\begin{abstract}
The approximation of parabolic equations with nonhomogeneous Dirichlet boundary data by a numerical method that consists of finite elements for the space discretization and the backward Euler time discretization is studied. The boundary values are assumed in a least squares sense. It is shown that this method achieves an optimal rate of convergence for rough (only $L^{2}$ ) boundary data and for smooth data as well. The results of numerical computations which confirm the robust theoretical error estimates are also presented.
\end{abstract}

\section{INTRODUCTION}

Consider the initial boundary value problem

$$
\begin{aligned}
y_{t}+A y=0 & \text { in } \Omega \times[0, T], \\
y=g & \text { on } \Gamma \times[0, T], \\
y(\cdot, 0)=v & \text { on } \Omega,
\end{aligned}
$$

where $\Omega$ is an open bounded convex polygonal domain in $\mathbf{R}^{2}$ with boundary $\Gamma$. We assume the elliptic operator

$$
A=-\sum_{i, j=1}^{2} \frac{\partial}{\partial x_{i}}\left(a_{i, j}(x) \frac{\partial}{\partial x_{j}}\right)
$$

has smooth coefficients, say $C^{2}(\Omega)$, and the $2 \times 2$ symmetric matrix with entries $a_{i, j}$ is uniformly positive definite on $\Omega$.

In this paper we are primarily concerned with rough boundary data $g$ which belong to either the space $L^{\infty}\left(0, T ; L^{2}(\Gamma)\right)$ or $L^{2}\left(0, T ; L^{2}(\Gamma)\right)$. This is typical of certain problems in control theory where the control $g$ has the bang-bang property (see [17]). As such our scheme for approximating the solution of (1) is a building block for solving these control problems.

For Neumann boundary control problems of parabolic type the finite element approximation has been analyzed by Winther [22]. For related time-optimal control problems see Knowles [16].

Received by the editor October 18, 1991 and, in revised form, December 23, 1991 and February 10, 1992.

1991 Mathematics Subject Classification. Primary 65N30.

Key words and phrases. Finite elements, parabolic equations, backward Euler method.

The first author's research was supported in part by the U.S. Army Research Office through grant number 28535-MA. 
Ground-breaking work on parabolic control problems with boundary Dirichlet control was done by Lasiecka $[17,18,19]$. Approximation by finite element methods of problem (1) was also considered by Lasiecka [17, 18] and Choudury [6]. One such method that is analyzed in $[18,6]$ uses piecewise linear elements in space which vanish on $\Gamma$. Optimal-order convergence is proved for the continuous time method in [17] and for a fully discrete scheme in [6]. This nonstandard approach, while optimal for rough $g$, is suboptimal for smooth $g$.

The scheme we propose and analyze is optimal for both rough and smooth boundary data. Our approximation $u$ consists of piecewise constants in time and finite elements in space, and assumes the boundary values in a least squares sense. Specifically, let $V_{k}$ denote the space of piecewise constant functions on a partition $0=t_{0}<t_{1}<\cdots<t_{N}=T$, where $t_{n}=n k$ and $k>0$ is the time step. Then $\psi \in V_{k}$ if $\psi=\sum_{j=1}^{N} \psi_{j} \chi_{I_{j}}$, where $\chi_{I_{j}}$ is the characteristic function of $I_{j}=\left(t_{j-1}, t_{j}\right]$. We denote by $V_{h}$ a finite element space on $\Omega$ with parameter $h$. In our estimates with rough boundary data we require

$$
k=c h^{2} \text {; }
$$

however, this restriction is not necessary in our analysis for smooth boundary data.

To define our scheme, we need to introduce some $L^{2}$ projections. Let

$$
V_{h}^{0}=\left\{\chi \in V_{h}: \chi=0 \text { on } \Gamma\right\}
$$

and $V_{h}(\Gamma)$ denote the restriction of $V_{h}$ to $\Gamma$. Define the $L^{2}$ projections $Q_{h}: L^{2}(\Gamma) \rightarrow V_{h}(\Gamma)$ and $P_{k}: L^{2}(0, T) \rightarrow V_{k}$, and set $Q=Q_{h} P_{k}=P_{k} Q_{h}$. We denote on $I_{n}$

$$
P_{k} w=P_{k}^{n} w=\frac{1}{k} \int_{I_{n}} w(t) d t .
$$

Our numerical method is as follows: Find $u \in V_{h} \otimes V_{k}$ such that

$$
\sum_{n=1}^{N}\left[\left(u^{n}-u^{n-1}, \phi\right)+k a\left(u^{n}, \phi\right)\right]=0, \quad \forall \phi \in V_{h}^{0} \otimes V_{k},
$$

with $u=Q g$ on $\Sigma=\Gamma \times(0, T], u^{0}=\mathscr{P}_{h}^{0} v$, where $u^{n}$ is the restriction of $u$ to $\Omega \times I_{n}, \mathscr{P}_{h}^{0}: L^{2}(\Omega) \rightarrow V_{h}$ is the $L^{2}$ projection,

$$
a(w, z)=\int_{\Omega}\left(\sum_{i, j=1}^{2} a_{i, j}(x) \frac{\partial w}{\partial x_{i}} \frac{\partial z}{\partial x_{j}}\right) d x
$$

and $(w, z)=\int_{\Omega} w z d x$. In Theorem 1 we will consider $v \in H^{-1 / 2}(\Omega)$, bounded linear functionals on $H^{1 / 2}(\Omega)$, and define $\mathscr{P}_{h}^{0}: H^{-1 / 2}(\Omega) \rightarrow V_{h}$ by

$$
v(\phi)=\left(\mathscr{P}_{h}^{0} v, \phi\right), \quad \phi \in V_{h} .
$$

Subsequently we denote the duality pairing for $v \in H^{-1 / 2}(\Omega)$ and $\phi \in H^{1 / 2}(\Omega)$ by $(v, \phi)$.

It is straightforward to demonstrate that (3) is uniquely solvable since for each $n=1,2, \ldots, N$

$$
\left(u^{n}-u^{n-1}, \phi\right)+k a\left(u^{n}, \phi\right)=0
$$


for all $\phi \in V_{h}^{0}$, and on $\Gamma$

$$
u^{n}=\frac{1}{k} \int_{I_{n}} Q_{h} g(\cdot, t) d t .
$$

This method is equivalent to backward Euler and is the simplest discontinuous Galerkin method (see, for example, [14]). For more general discontinuous Galerkin methods, see $[8,9,15]$. Observe that defining the approximate boundary data through interpolation would be inappropriate, since the data, $g$, is not continuous.

We will prove the following error estimates.

Theorem 1. There exists a constant $C$ independent of $h, u$, and $y$ such that if (2) holds then

$$
\|y-u\|_{L^{2}\left(0, T ; L^{2}(\Omega)\right)} \leq C h^{1 / 2}\left(\|g\|_{L^{2}(\Sigma)}+\|v\|_{H^{-1 / 2}(\Omega)}\right)
$$

for $v \in H^{-1 / 2}(\Omega)$ and $g \in L^{2}(\Sigma)$.

Theorem 2. For any $\varepsilon>0$ there exists a constant $C$ independent of $h, u$, and $y$ such that if (2) holds then

$$
\max _{0 \leq n \leq N}\left\|(y-u)\left(\cdot, t_{n}\right)\right\|_{L^{2}(\Omega)} \leq C h^{1 / 2-\varepsilon}\left(\|g\|_{L^{\infty}\left(0, T ; L^{2}(\Gamma)\right)}+\|v\|_{H^{1 / 2}(\Omega)}\right),
$$

where $v \in H^{1 / 2}(\Omega)$ and $g \in L^{\infty}\left(0, T ; L^{2}(\Gamma)\right)$.

We denote by $C$ a positive generic constant that is independent of $h, k$, and the data pair $(v, g)$.

A key ingredient in the proofs of these error estimates is the orthogonal decomposition of $V_{h}: V_{h}=V_{h}^{0} \oplus\left(V_{h}^{0}\right)^{\perp}$, where

$$
\left(V_{h}^{0}\right)^{\perp}=\left\{\phi \in V_{h}:(\phi, \chi)=0, \chi \in V_{h}^{0}\right\}
$$

or

$$
\left(V_{h}^{0}\right)^{\perp}=\left\{\phi \in V_{h}: a(\phi, \chi)=0, \chi \in V_{h}^{0}\right\} .
$$

Following Bramble, Pasciak, and Schatz [3], we refer to the latter choice of $\left(V_{h}^{0}\right)^{\perp}$ as discrete $A$-harmonic functions.

The outline of the remainder of the paper is as follows. In $\S 2$ we discuss problem (1) in our setting with $\Omega$ a convex polygonal domain. We present a weak formulation for this problem that is suitable for our analysis and obtain a priori estimates in $L^{2}\left(0, T ; L^{2}(\Omega)\right)$ and $L^{\infty}\left(0, T ; L^{2}(\Omega)\right)$. In $\S 3$ we state the approximation-theoretic and inverse properties of $V_{h}$ needed in the proofs of the error estimates. We also derive certain useful estimates for functions in $\left(V_{h}^{0}\right)^{\perp}$. In $\S 4$ we establish a stability estimate for the method and prove the $L^{2}$ error estimate. Section 5 is devoted to the $L^{\infty}$ error analysis. In $\S 6$ we sketch the proofs for optimal $L^{2}$ and $L^{\infty}$ error estimates when $y$ is smooth. Finally, in the last section we present some numerical experiments.

\section{REgULARITY}

Lasiecka proved in [17] that there exists a unique solution to (1) on a domain $\Omega$ with smooth boundary $\Gamma$ which satisfies an a priori $L^{\infty}$ in time estimate (see (21)). Lions and Magenes [20] proved similar results which were $L^{2}$ in time but still require a smooth boundary. We shall need both $L^{\infty}$ and $L^{2}$ in 
time estimates in the case where $\Omega$ is a convex polygon. Although we suspect such results are known to specialists in partial differential equations we could not find them in the literature. Therefore, we sketch the proofs of these and related results for use in later sections.

We denote by $H^{m}(\Omega)$ the usual Sobolev space of integer order $m \geq 0$ with norm $\|\cdot\|_{m}$. Note that $H^{0}(\Omega)=L^{2}(\Omega)$. Similarly, $H^{r}(\Gamma)$ denotes the Sobolev space of integer order $r \geq 0$ on $\Gamma$ with norm $|\cdot|_{r}$, and on $H^{0}(\Gamma)=L^{2}(\Gamma)$ the inner product is given by

$$
\langle w, z\rangle=\int_{\Gamma} w z d \sigma
$$

As usual, the Sobolev space of order one with functions that have trace equal to zero on $\Gamma$ is denoted $H_{0}^{1}(\Omega)$. Also, $H^{-1}(\Gamma)$ is the dual of $H^{1}(\Gamma)$, and $H^{-1}(\Omega)$ is the dual of $H^{1}(\Omega)$. For real $s$ the spaces $H^{s}(\Omega)$ and $H^{s}(\Gamma)$ are defined by interpolation.

We will have occasion to use the following norm interpolation inequality (see Proposition 2.3 in Chapter 1, $\S 2$ of [20] or [5, Theorem 3.2.3, p. 180])

$$
\|w\|_{\theta r+(1-\theta) s} \leq C\|w\|_{r}^{\theta}\|w\|_{s}^{1-\theta},
$$

where $0 \leq \theta \leq 1$ and $0 \leq r, s \leq 2$. Also, from Grisvard [13, Theorem 1.5.10] we have for $\varepsilon>0$ and $z \in H^{1}(\Omega)$ the inequality

$$
|z|_{0}^{2} \leq \frac{C}{\varepsilon}\|z\|_{1}^{2}+\varepsilon\|z\|_{0}^{2} .
$$

From this it follows, for $v \in H^{2}(\Omega)$, that

$$
\left|\frac{\partial v}{\partial \nu_{A}}\right|_{0}^{2} \leq \frac{C}{\varepsilon}\|v\|_{2}^{2}+\varepsilon\|v\|_{1}^{2}
$$

where

$$
\frac{\partial v}{\partial \nu_{A}}=\sum_{i, j=1}^{2} a_{i, j}(x) \frac{\partial v}{\partial x_{j}} \nu_{i}
$$

and $\nu=\left(\nu_{1}, \nu_{2}\right)$ is the unit outward normal to $\Gamma$. Applying (6) to the second term on the right side and then the arithmetic-geometric mean inequality, we obtain

$$
\left|\frac{\partial v}{\partial \nu_{A}}\right|_{0}^{2} \leq \frac{C}{\varepsilon}\|v\|_{2}^{2}+\varepsilon^{3}\|v\|_{0}^{2} .
$$

On $\mathscr{Q}=\Omega \times[0, T]$ let $H^{s, r}(\mathscr{Q})=L^{2}\left(0, T ; H^{s}(\Omega)\right) \cap H^{r}\left(0, T ; L^{2}(\Omega)\right)$ with norm

$$
\|w\|_{s, r}=\left(\int_{0}^{T}\|w(\cdot, t)\|_{s}^{2} d t+\int_{\Omega}\|w(x, \cdot)\|_{r,[0, T]}^{2} d x\right)^{1 / 2}
$$

where $\|\cdot\|_{r,[0, T]}$ denotes the norm on $H^{r}([0, T])$. Similarly, $H^{s, r}(\Sigma)=$ $L^{2}\left(0, T ; H^{s}(\Gamma)\right) \cap H^{r}\left(0, T ; L^{2}(\Gamma)\right)$, and the norm on $H^{s, r}(\Sigma)$ will be denoted by $|\cdot| s, r$.

The elliptic operator $A$ defined by $a(u, v)=(A u, v)$ for $v \in H_{0}^{1}(\Omega)$ satisfies (see Grisvard [13, Chapter 3])

$$
\|u\|_{2} \leq C\|A u\|_{0}
$$


where $\mathscr{D}(A)=H^{2}(\Omega) \cap H_{0}^{1}(\Omega)$. There is a continuous extension of $A$ to $L^{2}(\Omega)$ which we also denote by $A$ and which is defined by $(A u, v)=(u, A v)$ for $v \in H_{0}^{1}(\Omega)$. This operator has an orthonormal in $L^{2}(\Omega)$ basis of eigenfunctions $\left\{\phi_{m}\right\}_{m=1}^{\infty} \subset \mathscr{D}(A)$ and real eigenvalues $0<\lambda_{1} \leq \lambda_{2} \leq \lambda_{3} \leq \cdots$ such that

$$
a\left(\phi_{m}, v\right)=\lambda_{m}\left(\phi_{m}, v\right) \quad \forall v \in H_{0}^{1}(\Omega)
$$

with

$$
a\left(\phi_{m}, \phi_{l}\right)=\lambda_{m}\left(\phi_{m}, \phi_{l}\right)=\lambda_{m} \delta_{m l},
$$

where $\delta_{m l}$ denotes the Kronecker data (see Babuška and Osborn [1]).

Following Bramble and Thomée [4], we denote by $\dot{H}^{s}(\Omega)$ the subspace of $L^{2}(\Omega)$ consisting of functions $v$ such that

$$
\|v\|_{\dot{H}^{s}(\Omega)}=\left(\sum_{m=1}^{\infty} \lambda_{m}^{s} v_{m}^{2}\right)^{1 / 2}<\infty,
$$

where $v_{m}=\left(v, \phi_{m}\right)$. It follows, for $0 \leq s \leq 2$, that

$$
\|v\|_{\dot{H}^{s}(\Omega)}=\left\|A^{s / 2} v\right\|_{0},
$$

where $A^{\gamma}$ is defined by $A^{\gamma} u=\sum_{m=1}^{\infty} \lambda_{m}^{\gamma} u_{m} \phi_{m}$. We note that $\dot{H}^{2}(\Omega)=\mathscr{D}(A)$, $\dot{H}^{1}(\Omega)=H_{0}^{1}(\Omega)=\mathscr{D}\left(A^{1 / 2}\right), \dot{H}^{0}(\Omega)=L^{2}(\Omega)$, and $\dot{H}^{s}(\Omega)=H^{s}(\Omega)$ for $0 \leq s<\frac{1}{2}$. Also by the $K$-method of interpolation introduced by Lions and Peetre (see Butzer and Berens [5, p. 166]) one gets $\dot{H}^{s}(\Omega)=\mathscr{D}\left(A^{s / 2}\right)=$ $\left[H_{0}^{1}(\Omega), L^{2}(\Omega)\right]_{s}$ for $0 \leq s \leq 1$.

For $g=0$ the solution of (1) is given by

$$
E(t) v=\sum_{m=1}^{\infty} e^{-\lambda_{m} t} v_{m} \phi_{m}
$$

For smooth $\Gamma$ one has the well-known smoothing property for $t>0$ and $0 \leq l \leq s$ :

$$
\|E(t) v\|_{\dot{H}^{s}(\Omega)} \leq C t^{-(s-l) / 2}\|v\|_{\dot{H}^{\prime}(\Omega)}, \quad v \in \dot{H}^{l}(\Omega) .
$$

For a convex polygon $\Omega$ the solution $E(\cdot) v$ is only guaranteed to lie in $\mathscr{D}(A)=$ $\dot{H}^{2}(\Omega)$. It follows that (11) is valid for $0 \leq l \leq s \leq 2$, by the same proof as in [4].

To establish the solvability of (1), we need the Dirichlet map $D: L^{2}(\Gamma) \rightarrow$ $H^{1 / 2}(\Omega)$ defined by

$$
(D g, A \phi)=-\left\langle g, \frac{\partial \phi}{\partial \nu_{A}}\right\rangle \quad \forall \phi \in \mathscr{D}(A) .
$$

It is well known that $D$ is a bounded mapping.

We denote the solution to (1) with inhomogeneous right side $f, v=0$, and $g=0$ by

$$
B f=\int_{0}^{t} E(t-s) f(\cdot, s) d s .
$$

Using the bounded mapping $T: L^{2}(\Omega) \rightarrow \mathscr{D}(A)$, defined by

$$
a(T h, \phi)=(h, \phi) \quad \forall \phi \in H_{0}^{1}(\Omega),
$$


we can show by energy arguments that

$$
\|B f\|_{1,0} \leq C\|T f\|_{1,0} \text { and }\|B f\|_{0,0} \leq C\|T f\|_{0,0},
$$

as well as for the $E$ operator

$$
\|E(\cdot) v\|_{1,0} \leq C\|v\|_{0} \text { and }\|E(\cdot) v\|_{0,0} \leq C\|v\|_{-1} .
$$

Taking $f=A \eta$ in the previous inequalities for $B$, we obtain

$$
\|B A \eta\|_{1,0} \leq C\|\eta\|_{1,0} \text { and }\|B A \eta\|_{0,0} \leq C\|\eta\|_{0,0} .
$$

By interpolation (see Theorem 5.1 in Chapter 1 of [20]) it follows that $B A$ : $H^{s, 0}(\mathscr{Q}) \rightarrow H^{s, 0}(\mathscr{Q})$ and $E(\cdot): H^{s-1}(\Omega) \rightarrow H^{s, 0}(\mathscr{Q})$ are bounded maps for $0 \leq s \leq 1$.

We now turn to defining $y$ as a solution of the following very weak formulation of (1): Find $y$ defined on $\mathscr{Q}$ such that

$$
\int_{0}^{T}\left(y, w_{t}-A w\right) d t=\int_{0}^{T}\left\langle g, \frac{\partial w}{\partial \nu_{A}}\right\rangle d t-(v, w(\cdot, 0))
$$

for all $w \in H^{2,1}(\mathscr{Q}) \cap H_{0}^{1}(\Omega)$ with $w(\cdot, T)=0$, where $v$ and $g$ are given. We will specify appropriate spaces for $v, g$, and the solution $y$ in what follows. We note that uniqueness holds since the only solution for zero data is $y=0$ (choose $w_{t}-A w=y$ ). Moreover, formulation (13) is essentially the transposition procedure of Lions and Magenes [20, Chapter 4, §8] and will be the starting point for the error analysis of our method. We will obtain $L^{\infty}$ and $L^{2}$ (in time) a priori estimates in terms of the data.

Let $\left\{\eta^{n}\right\}_{n=1}^{\infty}$ be a sequence of infinitely differentiable functions which have compact support in $\Omega$ for all $t$, and let $\left\{v^{n}\right\}_{n=1}^{\infty} \subset C^{\infty}(\bar{\Omega})$. Take $z^{n}$ to be the solution of

$$
z_{t}^{n}+A z^{n}=-\eta_{t}^{n} \quad \text { on } \mathscr{Q}
$$

with $z^{n}=0$ on $\Gamma$ and $z^{n}(\cdot, 0)=v^{n}-\eta^{n}(\cdot, 0)$. Through integration by parts in both $t$ and $x$ it is easy to show for $w \in H^{2,1}(\mathscr{Q}) \cap H_{0}^{1}(\Omega), w(\cdot, T)=0$,

$$
\int_{0}^{T}\left(z^{n}, w_{t}-A w\right) d t=-\int_{0}^{T}\left(\eta^{n}, w_{t}\right) d t+\left(v^{n}, w(\cdot, 0)\right) .
$$

Setting $y^{n}=z^{n}+\eta^{n}$, we find

$$
\int_{0}^{T}\left(y^{n}, w_{t}-A w\right) d t=\int_{0}^{T}\left(\eta^{n}, A w\right) d t+\left(v^{n}, w(\cdot, 0)\right) .
$$

Moreover, $z^{n}$ is given by

$$
z^{n}=E(\cdot)\left(v^{n}-\eta^{n}(\cdot, 0)\right)-B \eta_{t}^{n}
$$

and using integration by parts on the second term, we obtain

$$
y^{n}=E(\cdot) v^{n}+A B \eta^{n} \text {. }
$$

Note that $A$ and $B$ commute on $L^{2}$. From the boundedness of the mappings $E(\cdot)$ and $B A$ it follows that

$$
\left\|y^{n}\right\|_{1 / 2,0} \leq C\left(\left\|v^{n}\right\|_{-1 / 2}+\left\|\eta^{n}\right\|_{1 / 2,0}\right) .
$$


Assuming $v^{n} \rightarrow v$ in $H^{-1 / 2}(\Omega)$ and $\eta^{n} \rightarrow D g$ in $H^{1 / 2,0}(\mathscr{Q})$, we have $y^{n} \rightarrow y$, and from (14) it follows that $y$ satisfies

$$
\int_{0}^{T}\left(y, w_{t}-A w\right) d t=\int_{0}^{T}(D g, A w) d t+(v, w(\cdot, 0))
$$

for all $w \in H^{2,1}(\mathscr{Q}) \cap H_{0}^{1}(\Omega)$ with $w(\cdot, T)=0$. Also, from (16) we have

$$
y=E(\cdot) v+A B D g \text {. }
$$

By the definition of $D g, y$ satisfies (13) and

$$
\|y\|_{1 / 2,0} \leq C\left(\|v\|_{-1 / 2}+|g|_{0,0}\right) \text {. }
$$

With the same argument as in [17] it follows that for $v \in H^{1 / 2}(\Omega), g \in$ $L^{\infty}\left(0, T ; L^{2}(\Gamma)\right)$ (hence, $D g \in L^{\infty}\left(0, T ; H^{1 / 2}(\Omega)\right)$ ), and $\varepsilon>0$,

$$
\|y\|_{L^{\infty}\left(0, T ; H^{1 / 2-\varepsilon}(\Omega)\right)} \leq C\left(\|v\|_{1 / 2}+\|g\|_{L^{\infty}\left(0, T ; L^{2}(\Gamma)\right)}\right) .
$$

Formula (19) and estimate (21) were obtained in [17] for the case where $\Gamma$ is smooth.

Inequalities (21) and (20) give estimates on the regularity of the solution in terms of the data. We establish a priori bounds in Propositions 1 and 2 for the approximate solution, $u$, using the same data norms as in (21) and (20).

Finally we state results for the following backward in time parabolic problem that will be used in our analysis. If $f \in L^{2}(\mathscr{Q})$, then

$$
\begin{aligned}
w_{t}-A w=f & \text { in } \Omega \times[0, T], \\
w=0 & \text { on } \Gamma \times[0, T], \\
w(\cdot, T)=0 & \text { on } \Omega
\end{aligned}
$$

has a unique solution $w \in H^{2,1}(\mathscr{Q}) \cap H_{0}^{1}(\Omega)$. It is not difficult to prove that

$$
\|w\|_{2,1} \leq C\|f\|_{0,0}
$$

and

$$
\|w(\cdot, 0)\|_{1} \leq C\|f\|_{0,0}
$$

\section{APPROXIMATION PROPERTIES}

In this section we give a precise definition of our finite element space. We also list the required approximation properties of various projection operators we use and present several key technical results.

Let $0<h<1$ and $V_{h}$ be the space of continuous piecewise linear functions relative to a quasiuniform triangulation $T^{h}$ of $\Omega$. That is, for some $\alpha$ (independent of $h$ ) each triangle $\tau \in T^{h}$ contains a disc of radius $\alpha h$ and is contained in a disc of radius $h$.

Define $P_{h}^{0}: L^{2}(\Omega) \rightarrow V_{h}^{0}$ to be the $L^{2}$ projection, and let $P_{h}^{1}: H^{1}(\Omega) \rightarrow V_{h}^{0}$ denote the elliptic projection:

$$
a\left(P_{h}^{1} v, \phi\right)=a(v, \phi) \quad \forall \phi \in V_{h}^{0} .
$$

We now list, for later reference, some well-known (see Ciarlet [7,§3.1]) properties of $V_{h}$ and $V_{h}^{0}$. For $z \in H^{s}(\Omega), 1 \leq s \leq 2$,

$$
\begin{gathered}
\inf _{\phi \in V_{h}}\left(\|\phi-z\|_{0}+h\|\phi-z\|_{1}\right) \leq C h^{s}\|z\|_{s}, \\
\left|\left(I-Q_{h}\right) z\right|_{0} \leq C h^{s-1 / 2}\|z\|_{s} .
\end{gathered}
$$


We also use the inverse properties for $\chi \in V_{h}$ :

$$
\begin{array}{cc}
\|\chi\|_{s} \leq C h^{-(s-l)}\|\chi\|_{l}, & 0 \leq l \leq s \leq 1, \\
|\chi|_{1 / 2} \leq C h^{-1 / 2+r}|\chi|_{r}, & 0 \leq r \leq \frac{1}{2} .
\end{array}
$$

Remark. Inequality (25) follows from (24) by the use of (7) with $\varepsilon=h^{-1}$.

Remark. Using a duality argument together with (25), one obtains

$$
\left|\left(I-Q_{h}\right) \frac{\partial z}{\partial \nu_{A}}\right|_{0} \leq C h^{1 / 2}\|z\|_{2}, \quad z \in H^{2}(\Omega) .
$$

Indeed, for $A w=0$ in $\Omega$ and $w=\partial z / \partial \nu_{A}$ on $\Gamma$, we have by (25) and elliptic estimates

$$
\left|\left(I-Q_{h}\right) \frac{\partial z}{\partial \nu_{A}}\right|_{0}=\left|\left(I-Q_{h}\right) w\right|_{0} \leq C h^{1 / 2}\|w\|_{1} \leq C h^{1 / 2}\left|\frac{\partial z}{\partial \nu_{A}}\right|_{1 / 2},
$$

from which (28) follows by the trace theorem.

For $V_{h}^{0}$ it is well known that, for $z \in H^{s}(\Omega) \cap H_{0}^{1}(\Omega)$ with $1 \leq s \leq 2$,

$$
\inf _{\phi \in V_{h}^{0}}\left(\|\phi-z\|_{0}+h\|\phi-z\|_{1}\right) \leq C h^{s}\|z\|_{s} .
$$

Remark. An immediate consequence of (29) and a standard duality argument is:

(30) $\left\|\left(I-P_{h}^{1}\right) z\right\|_{l} \leq C h^{s-l}\|z\|_{s}, \quad z \in H_{0}^{1}(\Omega) \cap H^{s}(\Omega), 0 \leq l \leq 1 \leq s \leq 2$.

From (30), the boundedness of $P_{h}^{0}$ in $L^{2}$, and the norm interpolation inequality (6) it follows that

$$
\left\|\left(I-P_{h}^{0}\right) z\right\|_{0} \leq C h^{s}\|z\|_{s}, \quad 0 \leq s \leq 2, \quad z \in \mathscr{D}(A) .
$$

By similar arguments it follows from (24) that

$$
\left\|\left(I-\mathscr{P}_{h}^{0}\right) z\right\|_{0} \leq C h^{s}\|z\|_{s}, \quad 0 \leq s \leq 2, \quad z \in H^{s}(\Omega) .
$$

Remark. We note that (25), (28), (30), (31), and (32) are valid for any space $V_{h}$ satisfying (24), (26), (27), and (29).

Finally we list two approximation properties of the space $V_{k}$ for a generic Hilbert space $H$ :

$$
\begin{gathered}
\left\|\left(I-P_{k}\right) z\right\|_{L^{1}(0, T ; H)} \leq C k\left\|z_{t}\right\|_{L^{1}(0, T ; H)}, \quad z \in H^{1}(0, T ; H), \\
\left\|P_{k} z\right\|_{L^{1}(0, T ; H)} \leq C\|z\|_{L^{1}(0, T ; H)}, \quad z \in L^{1}(0, T ; H) .
\end{gathered}
$$

We now prove three lemmas that involve the following splittings of an arbitrary $u \in V_{h}$ :

$$
u=u_{h}+u_{p} \text { and } u=u_{H}+u_{P},
$$

where $u_{p}=P_{h}^{0} u$ and $u_{P}=P_{h}^{1} u$. Note that

$$
\left(u_{h}, \chi\right)=0, \quad \chi \in V_{h}^{0},
$$

and

$$
a\left(u_{H}, \chi\right)=0, \quad \chi \in V_{h}^{0} .
$$

The first two lemmas are slight generalizations of some results of [3]. 
Lemma 1. Let $u \in V_{h}$, and suppose $z \in H^{2}(\Omega)$ satisfies $a(z, \phi)=0$ for all $\phi \in H_{0}^{1}(\Omega)$ and $z=u$ on $\Gamma$. Then

$$
\left\|z-u_{H}\right\|_{1} \leq C h^{-1 / 2}|u|_{0} .
$$

Proof. By (3.34) of [3], $u_{H}-z \in H_{0}^{1}(\Omega)$ satisfies

$$
\left\|u_{H}-z\right\|_{1} \leq C a^{1 / 2}\left(z-u_{H}, z-u_{H}\right) \leq C|u|_{1 / 2}
$$

and (37) follows by the inverse property (27).

The next result follows from Lemma 1 by the triangle inequality, elliptic estimates, and (27).

Lemma 2. For any $u \in V_{h}$,

$$
\left\|u_{H}\right\|_{1} \leq C h^{-1 / 2}|u|_{0} .
$$

Lemma 3. Suppose $u \in V_{h}$; then

$$
\left\|u_{h}\right\|_{s} \leq C h^{1 / 2-s}|u|_{0}, \quad 0 \leq s \leq 1 .
$$

Proof. Since $C_{0}^{\infty}(\Omega)$ is dense in $H^{p}(\Omega), p \leq \frac{1}{2}$ (see [12]), there exists $\left\{v^{n}\right\} \subset$ $C_{0}^{\infty}(\Omega)$ such that $\lim _{n \rightarrow \infty}\left\|v^{n}-u_{H}\right\|_{1 / 2}=0$. Noting that $u_{h}=\left(I-P_{h}^{0}\right) u_{H}$, we then find

$$
\left\|u_{h}\right\|_{0}=\lim _{n \rightarrow \infty}\left\|\left(I-P_{h}^{0}\right) v^{n}\right\|_{0} \leq C h^{1 / 2} \lim _{n \rightarrow \infty}\left\|v^{n}\right\|_{1 / 2}=C h^{1 / 2}\left\|u_{H}\right\|_{1 / 2} .
$$

As in the proof of Lemma 1, we have

$$
\left\|u_{H}\right\|_{1 / 2} \leq\left\|u_{H}-z\right\|_{1 / 2}+\|z\|_{1 / 2} .
$$

By the elliptic estimate $\|z\|_{1 / 2} \leq C|u|_{0}$ together with (37) we obtain (40) for $s=0$. For the case $s=1$ the result follows by the inverse property (26) and the case $s=0$. Now the general result follows by norm interpolation.

\section{4. $L^{2}$ estimates}

In this section we prove Theorem 1 . We assume that $v \in H^{-1 / 2}(\Omega)$ and $g \in L^{2}(\Sigma)$, and hence $y \in L^{2}\left(0, T ; H^{1 / 2}(\Omega)\right)$ satisfies (13) and (20).

An integral part of the proof is a particular stability estimate for the numerical scheme. The stability analysis of the method is of some interest, independent of its use in proving Theorem 1, and we begin by stating the main stability bound.

Proposition 1 ( $L^{2}$ stability). There exists a constant $C$ independent of $h, k$, and the data pair $(v, g)$ such that if (2) holds then for $0 \leq n \leq N$,

$$
\sum_{j=1}^{n}\left(\left\|u^{j}-u^{j-1}\right\|_{0}^{2}+k a\left(u^{j}, u^{j}\right)\right)+\left\|u^{n}\right\|_{0}^{2} \leq C h^{-1}\left(\|v\|_{-1 / 2}^{2}+|g|_{0,0}^{2}\right) .
$$

Before proving this stability result, we state and prove an auxiliary lemma.

Lemma 4. There exists a constant $C$ such that for $0 \leq n \leq N$,

$$
\sum_{j=1}^{n}\left(\left\|u_{p}^{j}-u_{p}^{j-1}\right\|_{0}^{2}+k a\left(u_{p}^{j}, u_{p}^{j}\right)\right)+\left\|u_{p}^{n}\right\|_{0}^{2} \leq C h^{-1}\left(\|v\|_{-1 / 2}^{2}+|g|_{0,0}^{2}\right) .
$$


Proof. For any $\phi \in V_{h}^{0}$ we have

$$
\left(u^{j}-u^{j-1}, \phi\right)+k a\left(u^{j}, \phi\right)=0 .
$$

Substituting $u=u_{h}+u_{p}$ and using (35), we have

$$
\left(u_{p}^{j}-u_{p}^{j-1}, \phi\right)+k a\left(u_{p}^{j}, \phi\right)=-k a\left(u_{h}^{j}, \phi\right) \text {. }
$$

Choosing $\phi=u_{p}^{j}$ and summing, we have

$$
\begin{gathered}
\sum_{j=1}^{n}\left[\frac{1}{2}\left\|u_{p}^{j}-u_{p}^{j-1}\right\|_{0}^{2}+k a\left(u_{p}^{j}, u_{p}^{j}\right)\right]+\frac{1}{2}\left\|u_{p}^{n}\right\|_{0}^{2} \\
=\frac{1}{2}\left\|u_{p}^{0}\right\|_{0}^{2}-\sum_{j=1}^{n} k a\left(u_{h}^{j}, u_{p}^{j}\right) .
\end{gathered}
$$

Using the arithmetic-geometric mean inequality on the right side yields

$$
\begin{aligned}
& \sum_{j=1}^{n}\left(\frac{1}{2}\left\|u_{p}^{j}-u_{p}^{j-1}\right\|_{0}^{2}+\frac{1}{2} k a\left(u_{p}^{j}, u_{p}^{j}\right)\right)+\frac{1}{2}\left\|u_{p}^{n}\right\|_{0}^{2} \\
& \quad \leq \frac{1}{2}\left\|u_{p}^{0}\right\|_{0}^{2}+\frac{1}{2} \sum_{j=1}^{n} k a\left(u_{h}^{j}, u_{h}^{j}\right) .
\end{aligned}
$$

Since $u_{p}^{0}=P_{h}^{0} \mathscr{P}_{h}^{0} v$, we have

$$
\left\|u_{p}^{0}\right\|_{0}=\sup _{\psi \in L^{2}(\Omega)} \frac{\left(\mathscr{P}_{h}^{0} v, P_{h}^{0} \psi\right)}{\|\psi\|_{0}} \leq \sup _{\psi \in L^{2}(\Omega)} \frac{\|v\|_{-1 / 2}\left\|P_{h}^{0} \psi\right\|_{1 / 2}}{\|\psi\|_{0}} \leq C h^{-1 / 2}\|v\|_{-1 / 2},
$$

where the last step follows from the inverse inequality (26).

By Lemma 3 and the fact that $Q$ is bounded it follows that

$$
\begin{aligned}
\sum_{j=1}^{n} k a\left(u_{h}^{j}, u_{h}^{j}\right) & =\sum_{j=1}^{n} \int_{I_{j}} a\left(u_{h}^{j}, u_{h}^{j}\right) d t \leq C h^{-1} \sum_{j=1}^{n} \int_{I_{j}}|Q g|_{0}^{2} d t \\
& \leq C h^{-1}\|g\|_{L^{2}\left(0, t_{n} ; L^{2}(\Gamma)\right)}^{2} \cdot \square
\end{aligned}
$$

Proof of Proposition 1. By the triangle inequality and Lemma 4 it suffices to consider

$$
\sum_{j=1}^{n}\left(\left\|u_{h}^{j}-u_{h}^{j-1}\right\|_{0}^{2}+k a\left(u_{h}^{j}, u_{h}^{j}\right)\right)+\left\|u_{h}^{n}\right\|_{0}^{2}
$$

Using the inverse inequality (26) and assumption (2), we have

$$
\sum_{j=1}^{n}\left(\left\|u_{h}^{j}-u_{h}^{j-1}\right\|_{0}^{2}+k a\left(u_{h}^{j}, u_{h}^{j}\right)\right)+\left\|u_{h}^{n}\right\|_{0}^{2} \leq C \sum_{j=0}^{n}\left\|u_{h}^{j}\right\|_{0}^{2} .
$$

The proof of Proposition 1 follows by an application of Lemma 3:

$$
\begin{aligned}
\sum_{j=1}^{n}\left\|u_{h}^{j}\right\|_{0}^{2} & \leq C\left(h \sum_{j=1}^{n}\left|u^{j}\right|_{0}^{2}+\left\|u^{0}\right\|_{0}^{2}\right) \\
& \leq C h^{-1}\left(\sum_{j=1}^{n} k\left|u^{j}\right|_{0}^{2}+\|v\|_{-1 / 2}^{2}\right) \\
& \leq C h^{-1}\left(\|g\|_{L^{2}\left(0, t_{n} ; L^{2}(\Gamma)\right)}^{2}+\|v\|_{-1 / 2}^{2}\right)
\end{aligned}
$$


We are now in a position to prove the main result of this section.

Proof of Theorem 1. Letting $w \in H^{2,1}(\mathscr{Q}) \cap H_{0}^{1}(\Omega)$ with $w(\cdot, T)=0$, we obtain

$$
\begin{aligned}
& \int_{0}^{T}\left(u, w_{t}-A w\right) d t=\sum_{n=1}^{N} \int_{I_{n}}\left[\left(u^{n}, w_{t}\right)-\left(u^{n}, A w\right)\right] d t \\
& \quad=\sum_{n=1}^{N} \int_{I_{n}}\left[k^{-1}\left(u^{n}, w^{n}-w^{n-1}\right)-a\left(u^{n}, w\right)+\left\langle Q g, \frac{\partial w}{\partial \nu_{A}}\right\rangle\right] d t
\end{aligned}
$$

We apply summation by parts to the first term:

$$
\begin{aligned}
& \sum_{n=1}^{N} \int_{I_{n}} k^{-1}\left(u^{n}, w^{n}-w^{n-1}\right) d t \\
& \quad=\left(u^{N}, w^{N}\right)-\left(u^{0}, w^{0}\right)-\sum_{n=1}^{N} \int_{I_{n}} k^{-1}\left(u^{n}-u^{n-1}, w^{n-1}\right) d t .
\end{aligned}
$$

But $w^{N}=w(\cdot, T)=0$, so we have

$$
\begin{aligned}
\int_{0}^{T}\left(u, w_{t}-A w\right) d t & \\
=\sum_{n=1}^{N} \int_{I_{n}}( & -k^{-1}\left(u^{n}-u^{n-1}, w^{n-1}\right) \\
& \left.-a\left(u^{n}, w\right)+\left\langle Q g, \frac{\partial w}{\partial \nu_{A}}\right\rangle\right) d t-\left(u^{0}, w^{0}\right) .
\end{aligned}
$$

Subtracting this from (13) and letting $w_{t}-A w=e$, where $e=y-u$, we have

$$
\begin{aligned}
\|e\|_{0,0}^{2}= & \sum_{n=1}^{N} \int_{I_{n}}\left(k^{-1}\left(u^{n}-u^{n-1}, w^{n-1}\right)+a\left(u^{n}, w\right)\right) d t \\
& +\sum_{n=1}^{N} \int_{I_{n}}\left\langle(I-Q) g, \frac{\partial w}{\partial \nu_{A}}\right\rangle d t-\left[(v, w(\cdot, 0))-\left(\mathscr{P}_{h}^{0} v, w(\cdot, 0)\right)\right] \\
= & J_{1}+J_{2}-J_{3} .
\end{aligned}
$$

We estimate the individual terms in reverse order. Clearly,

$$
\begin{aligned}
\left|J_{3}\right| & =\left|\left(v,\left(I-\mathscr{P}_{h}^{0}\right) w(\cdot, 0)\right)\right| \leq\|v\|_{-1 / 2}\left\|\left(I-\mathscr{P}_{h}^{0}\right) w(\cdot, 0)\right\|_{1 / 2} \\
& \leq C h^{1 / 2}\|v\|_{-1 / 2}\|w(\cdot, 0)\|_{1} \leq C h^{1 / 2}\|v\|_{-1 / 2}\|e\|_{0,0},
\end{aligned}
$$

where the last step follows from the parabolic estimate (23).

We estimate $J_{2}$ by the approximation properties of $P_{k}$ and $Q_{h}$, the interpolation inequality (8), and the parabolic estimate $(22)$ :

$$
\begin{aligned}
J_{2} & =\int_{0}^{T}\left\langle\left(I-P_{k}\right) g, \frac{\partial w}{\partial \nu_{A}}\right\rangle d t+\int_{0}^{T}\left\langle P_{k} g,\left(I-Q_{h}\right) \frac{\partial w}{\partial \nu_{A}}\right\rangle d t \\
& =\int_{0}^{T}\left\langle\left(I-P_{k}\right) g, \frac{\partial}{\partial \nu_{A}}\left(I-P_{k}\right) w\right\rangle d t+\int_{0}^{T}\left\langle P_{k} g,\left(I-Q_{h}\right) \frac{\partial w}{\partial \nu_{A}}\right\rangle d t .
\end{aligned}
$$


Applying the Cauchy-Schwarz inequality to this, we obtain the estimate

$$
\left|J_{2}\right| \leq C|g|_{0,0}\left(\left|\frac{\partial}{\partial \nu_{A}}\left(I-P_{k}\right) w\right|_{0,0}^{2}+\left|\left(I-Q_{h}\right) \frac{\partial w}{\partial \nu_{A}}\right|_{0,0}^{2}\right)^{1 / 2}
$$

Inequality $(8)$ yields

$$
\begin{aligned}
\left|\frac{\partial}{\partial \nu_{A}}\left(I-P_{k}\right) w\right|_{0,0}^{2} & \leq \frac{C}{\varepsilon}\left\|\left(I-P_{k}\right) w\right\|_{2,0}^{2}+\varepsilon^{3}\left\|\left(I-P_{k}\right) w\right\|_{0,0}^{2} \\
& \leq \frac{C}{\varepsilon}\|w\|_{2,0}^{2}+\varepsilon^{3} k^{2}\left\|w_{t}\right\|_{0,0}^{2} .
\end{aligned}
$$

Choosing $\varepsilon=k^{-1 / 2}$ gives

$$
\left|\frac{\partial}{\partial \nu_{A}}\left(I-P_{k}\right) w\right|_{0,0}^{2} \leq C k^{1 / 2}\|w\|_{2,1}^{2}
$$

Since

$$
\left|\left(I-Q_{h}\right) \frac{\partial w}{\partial \nu_{A}}\right|_{0,0} \leq C h^{1 / 2}\|w\|_{2,0},
$$

we obtain, from assumption (2) and estimate (22),

$$
\left|J_{2}\right| \leq C h^{1 / 2}\|w\|_{2,1}|g|_{0,0} \leq C h^{1 / 2}\|e\|_{0,0}|g|_{0,0} \text {. }
$$

Finally, we turn to the estimation of $J_{1}$. We have

$$
\begin{aligned}
J_{1} & =\sum_{n=1}^{N}\left(u^{n}-u^{n-1}, w^{n-1}\right)+k a\left(u^{n}, P_{k}^{n} w\right) \\
& =\sum_{n=1}^{N}\left(u^{n}-u^{n-1}, w^{n-1}-P_{h}^{1} P_{k}^{n} w\right)+k a\left(u^{n},\left(I-P_{h}^{1}\right) P_{k}^{n} w\right) .
\end{aligned}
$$

Application of the Cauchy-Schwarz inequality gives

$$
\left|J_{1}\right| \leq T_{1} \cdot T_{2}
$$

where

$$
T_{1}=\left(\sum_{n=1}^{N}\left(\left\|u^{n}-u^{n-1}\right\|_{0}^{2}+k a\left(u^{n}, u^{n}\right)\right)\right)^{1 / 2}
$$

and

$$
T_{2}=\left(\sum_{n=1}^{N}\left(\left\|w^{n-1}-P_{h}^{1} P_{k}^{n} w\right\|_{0}^{2}+k a\left(\left(I-P_{h}^{1}\right) P_{k}^{n} w,\left(I-P_{h}^{1}\right) P_{k}^{n} w\right)\right)\right)^{1 / 2}
$$

In view of Proposition 1 it suffices to estimate $T_{2}$. First we note that by (30)

$$
\begin{aligned}
\left\|w^{n-1}-P_{h}^{1} P_{k}^{n} w\right\|_{0} & \leq\left\|w^{n-1}-P_{k}^{n} w\right\|_{0}+\left\|\left(I-P_{h}^{1}\right) P_{k}^{n} w\right\|_{0} \\
& \leq\left\|w^{n-1}-P_{k}^{n} w\right\|_{0}+C h^{2}\left\|P_{k}^{n} w\right\|_{2},
\end{aligned}
$$

and again by (30)

$$
a\left(\left(I-P_{h}^{1}\right) P_{k}^{n} w,\left(I-P_{h}^{1}\right) P_{k}^{n} w\right) \leq C h^{2}\left\|P_{k}^{n} w\right\|_{2}^{2} .
$$


It is straightforward to show that

$$
\left\|w^{n-1}-P_{k}^{n} w\right\|_{0} \leq k^{1 / 2}\left\|w_{t}\right\|_{L^{2}\left(I_{n} ; L^{2}(\Omega)\right)}
$$

and

$$
\left\|P_{k}^{n} w\right\|_{2} \leq k^{-1 / 2}\|w\|_{L^{2}\left(I_{n} ; H^{2}(\Omega)\right)}
$$

Hence, by (2) and the previous estimates (49)-(52),

$$
\begin{aligned}
T_{2} & \leq C\left(\sum_{n=1}^{N}\left(h^{4}+k h^{2}\right)\left\|P_{k}^{n} w\right\|_{2}^{2}+k\left\|w_{t}\right\|_{L^{2}\left(I_{n} ; L^{2}(\Omega)\right)}^{2}\right)^{1 / 2} \\
& \leq C h\left(\sum_{n=1}^{N}\|w\|_{L^{2}\left(I_{n} ; H^{2}(\Omega)\right)}^{2}+\left\|w_{t}\right\|_{L^{2}\left(I_{n} ; L^{2}(\Omega)\right)}^{2}\right)^{1 / 2} \\
& \leq C h\|w\|_{2,1} .
\end{aligned}
$$

By the parabolic estimate (22) we have $\|w\|_{2,1} \leq C\|e\|_{0,0}$ and hence $T_{2} \leq$ $C h\|e\|_{0,0}$. Combining this with (48) and Proposition 1 completes the proof.

\section{5. $L^{\infty}$ ESTIMATES}

In this section we prove Theorem 2 as well as an $L^{\infty}$ stability estimate in time. We assume $v \in H^{1 / 2}(\Omega)$ and $g \in L^{\infty}\left(0, T ; L^{2}(\Gamma)\right)$, and hence $y \in L^{\infty}\left(0, T ; H^{1 / 2-\varepsilon}(\Omega)\right)$ satisfies (13) and (21). We will use the spectral properties of the discrete elliptic operator $A_{h}: V_{h}^{0} \rightarrow V_{h}^{0}$ defined by

$$
a(\chi, \phi)=\left(A_{h} \chi, \phi\right) \quad \forall \phi \in V_{h}^{0} .
$$

Let $\left\{\psi_{i}^{h}, \lambda_{i}^{h}\right\}_{i=1}^{N_{h}}$ be an orthonormal eigensystem for $A_{h}$, that is,

$$
a\left(\psi_{i}^{h}, \phi\right)=\lambda_{i}^{h}\left(\psi_{i}^{h}, \phi\right)
$$

for all $\phi \in V_{h}^{0}$ with $0<\lambda_{1}^{h} \leq \lambda_{2}^{h} \leq \cdots \leq \lambda_{N_{h}}^{h}$, where $N_{h}=\operatorname{dim}\left(V_{h}^{0}\right)$ and $\left(\psi_{i}^{h}, \psi_{j}^{h}\right)=\delta_{i j}$. In our analysis we will use the norm

$$
\|\| \chi \|_{s}^{2}=\sum_{i=1}^{N_{h}}\left(\chi, \psi_{i}^{h}\right)^{2}\left(\lambda_{i}^{h}\right)^{s}, \quad 0 \leq s \leq 1,
$$

where $\chi \in V_{h}^{0}$ is given by $\chi=\sum_{i=1}^{N_{h}}\left(\chi, \psi_{i}^{h}\right) \psi_{i}^{h}$. It is easy to see that

$$
\|\chi\|_{0}^{2}=\sum_{i=1}^{N_{h}}\left(\chi, \psi_{i}^{h}\right)^{2}=\|\| \chi \|_{0}^{2}
$$

and

$$
a(\chi, \chi)=\left(A_{h} \chi, \chi\right)=\|\| \chi \|_{1}^{2} .
$$

By interpolation, Bank and Dupont [2] prove there exist constants $C_{0}$ and $C_{1}$ so that

$$
C_{0}\|\chi\|_{s} \leq\|\| \chi\left\|_{s} \leq C_{1}\right\| \chi \|_{s}, \quad 0 \leq s \leq 1, \chi \in V_{h}^{0} .
$$

Since $A_{h}$ is symmetric positive definite, we may also define

$$
A_{h}^{q} \chi=\sum_{i=1}^{N_{h}}\left(\chi, \psi_{i}^{h}\right)\left(\lambda_{i}^{h}\right)^{q} \psi_{i}^{h}, \quad 0 \leq q \leq 1 .
$$


It follows that

$$
\left\|A_{h}^{s / 2} \chi\right\|_{0}^{2}=\left(A_{h}^{s} \chi, \chi\right)=\sum_{i=1}^{N_{h}}\left(\chi, \psi_{i}^{h}\right)^{2}\left(\lambda_{i}^{h}\right)^{s}=\|\| \chi \|_{s}^{2} .
$$

The following lemma is critical to our proof of Theorem 2.

Lemma 5. There exists a constant $C$ such that for $0 \leq q \leq 1$

$$
\left\|A_{h}^{q}\left(I+k A_{h}\right)^{-n}\right\| \leq C t_{n}^{-q} .
$$

Remark. The inequality (53) is a discrete version of a key semigroup inequality used in [6, 17].

Proof of Lemma 5. We first assume $0 \leq q<1$. We have

$$
\left\|A_{h}^{q}\left(I+k A_{h}\right)^{-n}\right\|=\sup _{\lambda \in \sigma\left(A_{h}\right)} f(\lambda)
$$

where $f(t)=t^{q}(1+k t)^{-n}$, and $\sigma\left(A_{h}\right)$ denotes the spectrum of $A_{h}$. A short calculation shows that on $[0, \infty)$ the absolute maximum of $f$ occurs at $\lambda^{*}=$ $q /(k(n-q))$. It follows that

$$
f(\lambda) \leq f\left(\lambda^{*}\right)=\left(\frac{q}{k(n-q)}\right)^{q}\left(1+\frac{q}{n-q}\right)^{-n}=q^{q}(n k)^{-q}\left(\frac{n}{n-q}\right)^{q-n}
$$

Thus,

$$
\left\|A_{h}^{q}\left(I+k A_{h}\right)^{-n}\right\| \leq q^{q}\left(\frac{1}{1-q / n}\right)^{q-n} t_{n}^{-q} \leq C_{q} t_{n}^{-q} .
$$

The analysis above is valid for $q=1$, except when $n=1$ and $f$ has no maximum. However, the estimate for this case is obvious.

The following lemma establishes the boundedness of the projection $\mathscr{P}_{h}^{0}$.

Lemma 6. There exists a constant $C$ such that for all $z \in H^{s}(\Omega)$

$$
\left\|\mathscr{P}_{h}^{0} z\right\|_{s} \leq C\|z\|_{s}, \quad 0 \leq s \leq 1 .
$$

Proof. Let $\mathscr{P}_{h}^{1}: H^{1}(\Omega) \rightarrow V_{h}$ satisfy

$$
\left(\mathscr{P}_{h}^{1} z, \phi\right)_{1}=(z, \phi)_{1}, \quad \phi \in V_{h},
$$

where $(\cdot, \cdot)_{1}$ denotes the inner product on $H^{1}(\Omega)$.

It follows by a standard duality argument that

$$
\left\|\left(I-\mathscr{P}_{h}^{1}\right) z\right\|_{0} \leq C h\|z\|_{1}, \quad z \in H^{1}(\Omega) .
$$

By definition, $\mathscr{P}_{h}^{0}$ is a bounded linear operator from $L^{2}(\Omega)$ into itself. Also we have

$$
\begin{aligned}
\left\|\mathscr{P}_{h}^{0} z\right\|_{1} & \leq\left\|\left(\mathscr{P}_{h}^{0}-\mathscr{P}_{h}^{1}\right) z\right\|_{1}+\left\|\mathscr{P}_{h}^{1} z\right\|_{1} \\
& \leq C h^{-1}\left(\left\|\left(I-\mathscr{P}_{h}^{0}\right) z\right\|_{0}+\left\|\left(I-\mathscr{P}_{h}^{1}\right) z\right\|_{0}\right)+\|z\|_{1} \\
& \leq C\|z\|_{1},
\end{aligned}
$$

where we used the approximation properties of $\mathscr{P}_{h}^{0}$ and (57) in the last estimate. Thus, $\mathscr{P}_{h}^{0}$ is a bounded map from $H^{1}(\Omega)$ into itself. By interpolation (see Theorem 5.1 in Chapter 1 of [20]), $\mathscr{P}_{h}^{0}: H^{s}(\Omega) \rightarrow H^{s}(\Omega)$ is bounded for $0 \leq$ $s \leq 1$.

We now give the $L^{\infty}$ in time stability estimate. 
Proposition 2 ( $L^{\infty}$ stability). Suppose $v \in H^{1 / 2}(\Omega)$ and $g \in L^{\infty}\left(0, T ; L^{2}(\Gamma)\right)$. Then for $0 \leq n \leq N$,

$$
\|u\|_{L^{\infty}\left(0, t_{n} ; H^{1}(\Omega)\right)} \leq C h^{-1 / 2}\left(\|v\|_{1 / 2}+\ln \left(\frac{1}{h}\right)\|g\|_{L^{\infty}\left(0, t_{n} ; L^{2}(\Gamma)\right)}\right) .
$$

Proof. For $\phi \in V_{h}^{0}$ we have

$$
\left(u_{p}^{n}-u_{p}^{n-1}, \phi\right)+k a\left(u_{p}^{n}, \phi\right)=-k a\left(u_{h}^{n}, \phi\right),
$$

and since $-a\left(u_{h}^{n}, \phi\right)=a\left(u_{p}^{n}-u_{P}^{n}, \phi\right)$, we obtain

$$
\left(I+k A_{h}\right) u_{p}^{n}=u_{p}^{n-1}+k A_{h}\left(u_{p}^{n}-u_{P}^{n}\right) .
$$

It now follows that

$$
u_{p}^{n}=\left(I+k A_{h}\right)^{-n} u_{p}^{0}+k \sum_{j=1}^{n}\left(I+k A_{h}\right)^{-j} A_{h}\left(u_{p}^{n-j+1}-u_{P}^{n-j+1}\right),
$$

and hence

$$
\begin{aligned}
\left\|u_{p}^{n}\right\|_{1} \leq & \left\|\left(I+k A_{h}\right)^{-n} A_{h}^{1 / 4}\right\|\left\|u_{p}^{0}\right\|_{1 / 2} \\
& +k \sum_{j=1}^{n}\left\|A_{h}\left(I+k A_{h}\right)^{-j}\right\|\left\|u_{p}^{n-j+1}-u_{P}^{n-j+1}\right\|_{1} .
\end{aligned}
$$

By Lemma 5 it follows that

$$
\left\|u_{p}^{n}\right\|_{1} \leq C t_{n}^{-1 / 4}\left\|u_{p}^{0}\right\|_{1 / 2}+C k \sum_{j=1}^{n} t_{j}^{-1} \mid\left\|u_{p}^{n-j+1}-u_{P}^{n-j+1}\right\|_{1} .
$$

Now we estimate the terms $\left\|u_{p}^{0}\right\|_{1 / 2}$ and $\left\|\mid u_{p}^{j}-u_{P}^{j}\right\|_{1}$. We have by norm equivalence

$$
\left\|u_{p}^{0}\right\|_{1 / 2} \leq C\left\|u_{p}^{0}\right\|_{1 / 2}=C\left\|P_{h}^{0} \mathscr{P}_{h}^{0} v\right\|_{1 / 2} \leq C\left(\left\|\left(I-P_{h}^{0}\right) \mathscr{P}_{h}^{0} v\right\|_{1 / 2}+\left\|\mathscr{P}_{h}^{0} v\right\|_{1 / 2}\right) .
$$

By Lemma 6, the inverse property (26), and Lemma 3 we find

$$
\left\|\mid u_{p}^{0}\right\|\left\|_{1 / 2} \leq C\left(h^{-1 / 2}\left\|\left(I-P_{h}^{0}\right) \mathscr{P}_{h}^{0} v\right\|_{0}+\|v\|_{1 / 2}\right) \leq C\right\| v \|_{1 / 2} .
$$

Also, since $u_{p}^{m}-u_{P}^{m}=u_{H}^{m}-u_{h}^{m}$, the triangle inequality and Lemmas 2 and 3 imply that

$$
||\left|u_{p}^{m}-u_{P}^{m}\right|||_{1} \leq C h^{-1 / 2}\left|u^{m}\right|_{0} \leq C h^{-1 / 2}|g|_{L^{\infty}\left(I_{m} ; L^{2}(\Gamma)\right)} .
$$

Combining these estimates gives

$$
\left\|u_{p}\right\|_{L^{\infty}\left(0, t_{n} ; H^{1}(\Omega)\right)} \leq C h^{-1 / 2}\left(\|v\|_{1 / 2}+\ln \left(\frac{1}{h}\right)\|g\|_{L^{\infty}\left(0, t_{n} ; L^{2}(\Gamma)\right)}\right),
$$

since $\sum_{j=1}^{n} k t_{j}^{-1} \leq C \ln \left(\frac{1}{h}\right)$. It is not difficult, using Lemma 3, to show that

$$
\left\|u_{h}\right\|_{L^{\infty}\left(0, t_{n} ; L_{2}(\Omega)\right)} \leq C h^{1 / 2}\|g\|_{L^{\infty}\left(0, t_{n} ; L^{2}(\Gamma)\right)}
$$

and

$$
\max _{0 \leq t \leq t_{n}} a\left(u_{h}(\cdot, t), u_{h}(\cdot, t)\right) \leq C h^{-1 / 2}\|g\|_{L^{\infty}\left(0, t_{n} ; L^{2}(\Gamma)\right)} .
$$

Finally, by the triangle inequality, we combine (61), (62), and (63) to give (58). 
We complete this section by giving the proof of Theorem 2 .

Proof of Theorem 2. By the triangle inequality,

$$
\|(u-y)(\cdot, T)\|_{0} \leq\left\|\left(u-\mathscr{P}_{h}^{0} y\right)(\cdot, T)\right\|_{0}+\left\|\left(I-\mathscr{P}_{h}^{0}\right) y(\cdot, T)\right\|_{0} .
$$

Letting $\eta=\left(u-\mathscr{P}_{h}^{0} y\right)(\cdot, T)$ and using the approximation properties of $\mathscr{P}_{h}^{0}$ along with the a priori estimate $(21)$, we have

$$
\|(u-y)(\cdot, T)\|_{0} \leq\|\eta\|_{0}+C h^{1 / 2-\varepsilon}\left(\|v\|_{1 / 2}+\|g\|_{L^{\infty}\left(0, T ; L^{2}(\Gamma)\right)}\right) .
$$

Thus, we must estimate $\eta$. Let $w \in H^{2,1}(\mathscr{Q}) \cap H_{0}^{1}(\Omega)$ satisfy $w_{t}-A w=0$ and $w(\cdot, T)=\eta$. Starting with (13), the discrete equations for $u^{n}$, and summation by parts, we obtain

$$
\begin{aligned}
((u-y)(\cdot, T), \eta)= & \sum_{n=1}^{N} \int_{I_{n}}\left(k^{-1}\left(u^{n}-u^{n-1}, w^{n-1}\right)+a\left(u^{n}, w\right)\right) d t \\
& +\sum_{n=1}^{N} \int_{I_{n}}\left\langle(I-Q) g, \frac{\partial w}{\partial \nu_{A}}\right\rangle d t-\left(\left(I-\mathscr{P}_{h}^{0}\right) v, w(\cdot, 0)\right) .
\end{aligned}
$$

Adding and subtracting $\mathscr{P}_{h}^{0} y(\cdot, T)$ on the left side, we have, since

$$
\begin{gathered}
\left(\left(I-\mathscr{P}_{h}^{0}\right) y(\cdot, T), \eta\right)=0 \\
\|\eta\|_{0}^{2}=\sum_{n=1}^{N} \int_{I_{n}}\left(k^{-1}\left(u^{n}-u^{n-1}, w^{n-1}\right)+a\left(u^{n}, w\right)\right) d t \\
+\int_{0}^{T}\left\langle(I-Q) g, \frac{\partial w}{\partial \nu_{A}}\right\rangle d t-\left(\left(I-\mathscr{P}_{h}^{0}\right) v, w(\cdot, 0)\right) \\
=S_{1}+S_{2}+S_{3} .
\end{gathered}
$$

We now estimate the terms $S_{j}$. For the last we have

$$
\left|S_{3}\right| \leq\left\|\left(I-\mathscr{P}_{h}^{0}\right) v\right\|_{0}\|w(\cdot, 0)\|_{0} \leq C h^{1 / 2}\|v\|_{1 / 2}\|\eta\|_{0},
$$

where the estimate for $w(\cdot, 0)$ follows from $(23)$. We now estimate $S_{2}$ :

$$
S_{2}=\int_{0}^{T}\left\langle g,\left(I-P_{k}\right) \frac{\partial w}{\partial \nu_{A}}\right\rangle d t+\int_{0}^{T}\left\langle P_{k} g,\left(I-Q_{h}\right) \frac{\partial w}{\partial \nu_{A}}\right\rangle d t
$$

By the Cauchy-Schwarz and Hölder inequalities we have

$$
\begin{aligned}
&\left|S_{2}\right| \leq\|g\|_{L^{\infty}\left(0, T ; L^{2}(\Gamma)\right)}\left(\left\|\left(I-P_{k}\right) \frac{\partial w}{\partial \nu_{A}}\right\|_{L^{1}\left(0, T ; L^{2}(\Gamma)\right)}\right. \\
&\left.+\left\|\left(I-Q_{h}\right) \frac{\partial w}{\partial \nu_{A}}\right\|_{L^{1}\left(0, T ; L^{2}(\Gamma)\right)}\right) .
\end{aligned}
$$

We estimate the terms on the right side of (67) separately. Using (8), we have

$$
\begin{aligned}
\|(I- & \left.P_{k}\right) \frac{\partial w}{\partial \nu_{A}}\left\|_{L^{1}\left(0, T ; L^{2}(\Gamma)\right)}=\right\| \frac{\partial}{\partial \nu_{A}}\left(I-P_{k}\right) w \|_{L^{1}\left(0, T ; L^{2}(\Gamma)\right)} \\
& \leq C \varepsilon^{-1 / 2}\left\|\left(I-P_{k}\right) w\right\|_{L^{1}\left(0, T ; H^{2}(\Omega)\right)}+\varepsilon^{3 / 2}\left\|\left(I-P_{k}\right) w\right\|_{L^{1}\left(0, T ; L^{2}(\Omega)\right)} .
\end{aligned}
$$


Choosing $\varepsilon=k^{-1 / 2}$, we obtain

$$
\begin{aligned}
\|(I- & \left.P_{k}\right) \frac{\partial w}{\partial \nu_{A}}\|\|_{L^{1}\left(0, T ; L^{2}(\Gamma)\right)} \\
& \leq C k^{1 / 4}\left\|\left(I-P_{k}\right) w\right\|_{L^{1}\left(0, T ; H^{2}(\Omega)\right)}+k^{-3 / 4}\left\|\left(I-P_{k}\right) w\right\|_{L^{1}\left(0, T ; L^{2}(\Omega)\right)} .
\end{aligned}
$$

Since $w_{t}=-A w$, we have

$$
\left\|\left(I-P_{k}\right) w\right\|_{L^{1}\left(0, T ; L^{2}(\Omega)\right)} \leq C k\left\|w_{t}\right\|_{L^{1}\left(0, T ; L^{2}(\Omega)\right)} \leq C k\|w\|_{L^{1}\left(0, T ; H^{2}(\Omega)\right)}
$$

and

so that

$$
\left\|\left(I-P_{k}\right) w\right\|_{L^{1}\left(0, T ; H^{2}(\Omega)\right)} \leq C\|w\|_{L^{1}\left(0, T ; H^{2}(\Omega)\right)},
$$

$$
\left\|\left(I-P_{k}\right) \frac{\partial w}{\partial \nu_{A}}\right\|_{L^{1}\left(0, T ; L^{2}(\Gamma)\right)} \leq C k^{1 / 4}\|w\|_{L^{1}\left(0, T ; H^{2}(\Omega)\right)} .
$$

To complete the estimation of $S_{2}$, we turn to the last term in (67). By (28) we obtain

$$
\left\|\left(I-Q_{h}\right) \frac{\partial w}{\partial \nu_{A}}\right\|_{L^{1}\left(0, T ; L^{2}(\Gamma)\right)} \leq C h^{1 / 2}\|w\|_{L^{1}\left(0, T ; H^{2}(\Omega)\right)} .
$$

Combining estimates (68) and (69) in (67), we have

$$
\left|S_{2}\right| \leq C\left(k^{1 / 4}+h^{1 / 2}\right)\|g\|_{L^{\infty}\left(0, T ; L^{2}(\Gamma)\right)}\|w\|_{L^{1}\left(0, T ; H^{2}(\Omega)\right)} .
$$

We now apply the estimate (11) with $s=2$ and $l=\varepsilon$ :

(70) $\|w\|_{L^{1}\left(0, T ; H^{2}(\Omega)\right)} \leq C \int_{0}^{T}(T-t)^{-(2-\varepsilon) / 2}\|\eta\|_{\varepsilon} d t \leq C_{T}\|\eta\|_{\varepsilon} \leq C_{T} h^{-\varepsilon}\|\eta\|_{0}$,

where we used the inverse inequality (26) in the last step on $\eta \in V_{h}$. Thus,

$$
\left|S_{2}\right| \leq C h^{1 / 2-\varepsilon}\|g\|_{L^{\infty}\left(0, T ; L^{2}(\Gamma)\right)}\|\eta\|_{0} .
$$

Finally, we turn to estimating $S_{1}$. From (3) we have for any $\phi \in V_{h}^{0} \otimes V_{k}$

$$
\begin{aligned}
S_{1}= & \sum_{n=1}^{N}\left(\left(u^{n}-u^{n-1}, w^{n-1}-\phi\right)+k a\left(u^{n}, P_{k}^{n} w-\phi\right)\right) \\
= & \sum_{n=1}^{N}\left(\left(u_{p}^{n}-u_{p}^{n-1}, P_{h}^{0} w^{n-1}-\phi\right)+k a\left(u_{P}^{n}, P_{h}^{1} P_{k}^{n} w-\phi\right)\right) \\
& +\sum_{n=1}^{N}\left(\left(u_{h}^{n}-u_{h}^{n-1},\left(I-P_{h}^{0}\right) w^{n-1}\right)+k a\left(u_{H}^{n},\left(I-P_{h}^{1}\right) P_{k}^{n} w\right)\right) .
\end{aligned}
$$

The choice $\phi=P_{h}^{0} w^{n-1}$ on $I_{n}$ gives

$$
\begin{aligned}
S_{1}= & \sum_{n=1}^{N} k a\left(u_{P}^{n}, P_{h}^{1} P_{k}^{n} w-P_{h}^{0} w^{n-1}\right) \\
& +\sum_{n=1}^{N}\left[\left(u_{h}^{n}-u_{h}^{n-1},\left(I-P_{h}^{0}\right) w^{n-1}\right)+k a\left(u_{H}^{n},\left(I-P_{h}^{1}\right) P_{k}^{n} w\right)\right] \\
= & T_{1}-T_{2} .
\end{aligned}
$$


We estimate the terms $T_{1}$ and $T_{2}$. By the Cauchy-Schwarz and Hölder inequalities we have

$$
\left|T_{1}\right| \leq\|u\|_{L^{\infty}\left(0, T ; H^{1}(\Omega)\right)} \sum_{n=1}^{N} k\left\|P_{h}^{1} P_{k}^{n} w-P_{h}^{0} w^{n-1}\right\|_{1} .
$$

By the triangle inequality and inverse property (26),

$$
\begin{aligned}
\left\|P_{h}^{1} P_{k}^{n} w-P_{h}^{0} w\right\|_{1} \leq & \left\|\left(I-P_{h}^{1}\right) P_{k}^{n} w\right\|_{1} \\
& +C h^{-1}\left(\left\|\left(I-P_{h}^{0}\right) P_{k}^{n} w\right\|_{0}+\left\|P_{h}^{0}\left(P_{k}^{n} w-w^{n-1}\right)\right\|_{0}\right),
\end{aligned}
$$

and hence by (30), (31), and the boundedness of $P_{h}^{0}$,

$$
\left\|P_{h}^{1} P_{k}^{n} w-P_{h}^{0} w^{n-1}\right\|_{1} \leq C\left(h\left\|P_{k}^{n} w\right\|_{2}+h^{-1}\left\|P_{k}^{n} w-w^{n-1}\right\|_{0}\right) .
$$

It is not difficult to show

$$
\left\|P_{k}^{n} w\right\|_{2} \leq k^{-1}\|w\|_{L^{1}\left(I_{n} ; H^{2}(\Omega)\right)}
$$

and

$$
\left\|P_{k}^{n} w-w^{n-1}\right\|_{0} \leq\left\|w_{t}\right\|_{L^{1}\left(I_{n} ; L^{2}(\Omega)\right)} .
$$

Combining these estimates, we obtain

$$
\begin{aligned}
& \sum_{n=1}^{N} k\left\|P_{h}^{1} P_{k}^{n} w-P_{h}^{0} w^{n-1}\right\|_{1} \\
& \quad \leq C \sum_{n=1}^{N} h\|w\|_{L^{1}\left(I_{n} ; H^{2}(\Omega)\right)}+k h^{-1}\left\|w_{t}\right\|_{L^{1}\left(I_{n} ; L^{2}(\Omega)\right)} \\
& \quad \leq C h\|w\|_{L^{1}\left(0, T ; H^{2}(\Omega)\right)}
\end{aligned}
$$

where we used (2) and $w_{t}=-A w$ in the last step. By (70), (72), (76), and Proposition 2 we complete the estimate of $T_{1}$ :

$$
\left|T_{1}\right| \leq C h^{1 / 2-\varepsilon}\left(\|v\|_{1 / 2}+\ln \left(\frac{1}{h}\right)\|g\|_{L^{\infty}\left(0, T ; L^{2}(\Gamma)\right)}\right)\|\eta\|_{0} .
$$

For $T_{2}$ we have $\left|T_{2}\right| \leq F_{1} \cdot F_{2}$, where

$$
F_{1}=\left\|u_{h}\right\|_{L^{\infty}\left(0, T ; L^{2}(\Omega)\right)}+h\left\|u_{H}\right\|_{L^{\infty}\left(0, T ; L^{2}(\Omega)\right)} \leq C h^{1 / 2}\|g\|_{L^{\infty}\left(0, T ; L^{2}(\Omega)\right)}
$$

by Lemmas 2 and 3. Moreover (since $P_{k}^{n} w^{n-1}=w^{n-1}$ ),

$$
\begin{aligned}
F_{2} & =\sum_{n=1}^{N}\left\|\left(I-P_{h}^{0}\right) P_{k}^{n} w^{n-1}\right\|_{0}+k h^{-1}\left\|\left(I-P_{h}^{1}\right) P_{k}^{n} w\right\|_{1} \\
& \leq \sum_{n=1}^{N}\left\|\left(I-P_{h}^{0}\right) P_{k}^{n}\left(w^{n-1}-w\right)\right\|_{0}+\left\|\left(I-P_{h}^{0}\right) P_{k}^{n} w\right\|_{0}+k h^{-1}\left\|\left(I-P_{h}^{1}\right) P_{k}^{n} w\right\|_{1} \\
& \leq C \sum_{n=1}^{N}\left\|w^{n-1}-P_{k}^{n} w\right\|_{0}+\left(h^{2}+k\right)\left\|P_{k}^{n} w\right\|_{2},
\end{aligned}
$$

where we used (30) and (31) in the last step. The last estimate together with (74), (75), and assumption (2) completes the estimation of $F_{2}$ :

$$
F_{2} \leq C\|w\|_{L^{1}\left(0, T ; H^{2}(\Omega)\right)} .
$$


Combining the estimates for $F_{1}$ and $F_{2}$, and using (70) gives

$$
\left|T_{2}\right| \leq C h^{1 / 2-\varepsilon}\|g\|_{L^{\infty}\left(0, T ; L^{2}(\Gamma)\right)}\|\eta\|_{0} \text {. }
$$

This completes the estimation of $S_{1}$. Combining the estimates for $S_{1}, S_{2}, S_{3}$ in (65) gives the required bound for $\|\eta\|_{0}$. Substituting this in (64), we obtain the required estimate at $t_{N}=T$. Since the same argument applies for any $t_{n}$, the proof of Theorem 2 is complete.

\section{SMOоTH SOlUtion ESTIMATES}

In this section we show that an optimal-order convergence rate is obtained for our method when $y \in H^{2,1}(\mathscr{Q})$. Our analysis is quite similar to that contained in $[14, \S 8.4 ; 15]$. Note that there are no restrictions on $k$ or $h$ in this section. As usual, the error is split into two components:

$$
e=y-u=y-\tilde{u}+\tilde{u}-u=\theta-\eta,
$$

where $\tilde{u} \in V_{h} \otimes V_{k}$ is defined on $I_{n}$ as follows:

$$
\tilde{u}=\tilde{u}^{n}=\frac{1}{k} \int_{I_{n}} \hat{u}(\cdot, s) d s, \quad n>0,
$$

and $\tilde{u}^{0}=u^{0}$. The function $\hat{u}(\cdot, t) \in V_{h}$ is an elliptic projection:

$$
a((\hat{u}-y)(\cdot, t), \phi)=0, \quad \phi \in V_{h}^{0},
$$

for $0 \leq t \leq T$ and $\hat{u}=Q_{h} g$ on $\Gamma$. The following lemma gives the approximation properties of $\hat{u}$.

Lemma 7. There exists a constant $C$ independent of $h, \hat{u}$, and $y$ such that

$$
\|(y-\hat{u})(\cdot, t)\|_{0} \leq C h^{2}\|y(\cdot, t)\|_{2} .
$$

Proof. Define $\mathscr{P}_{h}: H^{1}(\Omega) \rightarrow V_{h}$ by the equation

$$
a\left(\mathscr{P}_{h} w, \chi\right)+h^{-1}\left\langle\mathscr{P}_{h} w, \chi\right\rangle=a(w, \chi)+h^{-1}\langle w, \chi\rangle
$$

for all $\chi \in V_{h}$. For $\xi \in H^{1}(\Omega)$ define the norm

$$
\mathscr{N}(\xi)=\left(a(\xi, \xi)+h^{-1}|\xi|_{0}^{2}\right)^{1 / 2} .
$$

It follows that for $w \in H^{2}(\Omega)$

$$
\mathscr{N}\left(\left(I-\mathscr{P}_{h}\right) w\right)=\inf _{\phi \in V_{h}} \mathscr{N}(\phi-w) \leq C h\|w\|_{2},
$$

where the last estimate follows from (7) and (30) by choosing $\varepsilon=h^{-1 / 2}$ and $\phi=P_{h}^{1} w$.

We note that $a\left(\mathscr{P}_{h} y, \phi\right)=a(y, \phi)$ for $\phi \in V_{h}^{0}$. Thus, $a\left(\hat{u}-\mathscr{P}_{h} y, \phi\right)=0$ for all $\phi \in V_{h}^{0}$. So, $\hat{u}-\mathscr{P}_{h} y$ is discrete $A$-harmonic and therefore by Lemma 2

$$
\begin{aligned}
a\left(\hat{u}-\mathscr{P}_{h} y, \hat{u}-\mathscr{P}_{h} y\right) & \leq C h^{-1}\left|\hat{u}-\mathscr{P}_{h} y\right|_{0}^{2} \\
& \leq C h^{-1}\left(\left|\left(I-Q_{h}\right) y\right|_{0}^{2}+\left|\left(I-\mathscr{P}_{h}\right) y\right|_{0}^{2}\right) \\
& \leq C h^{-1}\left|\left(I-\mathscr{P}_{h}\right) y\right|_{0}^{2} .
\end{aligned}
$$

This implies by the triangle inequality

$$
\mathscr{N}(\hat{u}-y) \leq C \mathscr{N}\left(\left(I-\mathscr{P}_{h}\right) y\right) .
$$


From this we obtain the optimal estimates

$$
a(\hat{u}-y, \hat{u}-y) \leq C h^{2}\|y\|_{2}^{2}
$$

and

$$
|\hat{u}-y|_{0}^{2} \leq C h^{3}\|y\|_{2}^{2} .
$$

Let $w \in \mathscr{D}(A)$ satisfy $A w=\hat{u}-y$ on $\Omega$. Then for any $\phi \in V_{h}^{0}$

$$
\begin{aligned}
\|\hat{u}-y\|_{0}^{2} & =a(\hat{u}-y, w-\phi)+\left\langle\left(I-Q_{h}\right) y,\left(I-Q_{h}\right) \frac{\partial w}{\partial \nu_{A}}\right\rangle \\
& \leq \mathcal{N}(\hat{u}-y)\left(a(w-\phi, w-\phi)+h\left|\left(I-Q_{h}\right) \frac{\partial w}{\partial \nu_{A}}\right|_{0}^{2}\right)^{1 / 2} \\
& \leq C h\|w\|_{2} \mathscr{N}(\hat{u}-y),
\end{aligned}
$$

where we chose $\phi=P_{h}^{1} w$ on the last step. Thus, by elliptic regularity,

$$
\|\hat{u}-y\|_{0} \leq \operatorname{ChN}(\hat{u}-y) .
$$

Combining this with the previous estimates proves (77).

Remark. The function $\hat{u}$ and the argument used in the proof of Lemma 7 were introduced in French and King [11]. Also, see the related work in Fix, Gunzburger, and Peterson [10].

By standard arguments we can now show, using the result of Lemma 7 ,

$$
\begin{gathered}
\|\theta\|_{0,0} \leq C\left(k+h^{2}\right)\|y\|_{2,1}, \\
\sum_{n=1}^{N} k\left\|\theta^{n}\right\|_{0}^{2} \leq C\left(k+h^{2}\right)\|y\|_{2,1},
\end{gathered}
$$

and

$$
\|\theta\|_{L^{\infty}\left(0, t_{n} ; L^{2}(\Omega)\right)} \leq C\left(k+h^{2}\right)\|y\|_{L^{\infty}\left(0, T ; H^{2}(\Omega)\right)},
$$

where $\theta^{n}=y\left(\cdot, t_{n}\right)-\tilde{u}^{n}$. In contrast to our analysis in $\S \S 4$ and 5 , the solution $y$ satisfies the following weak form for $\phi \in V_{h}^{0}$ :

$$
\int_{I_{n}}\left(k^{-1}\left(y^{n}-y^{n-1}, \phi\right)+a(y, \phi)\right) d t=0 .
$$

We are now in a position to state and prove the $L^{2}$ convergence theorem.

Theorem 3. If $y \in H^{2,1}(\mathscr{Q})$, then there exists a constant $C$ independent of $h, u$, and $y$ such that

$$
\|y-u\|_{0,0} \leq C\left(h^{2}+k\right)\|y\|_{2,1} .
$$

Proof. Define $z \in V_{h}^{0} \otimes V_{k}$, so that $z^{N}=0$ and

$$
k^{-1}\left(z^{n}-z^{n-1}, \phi\right)-a\left(z^{n-1}, \phi\right)=\left(\eta^{n}, \phi\right), \quad \phi \in V_{h}^{0} .
$$


Letting $\phi=\eta^{n}$ and integrating in time, we have

$$
\begin{aligned}
\|\eta\|_{0,0}^{2}= & \sum_{n=1}^{N} \int_{I_{n}}\left(k^{-1}\left(z^{n}-z^{n-1}, \eta^{n}\right)-a\left(z^{n-1}, \eta^{n}\right)\right) d t \\
= & \sum_{n=1}^{N} \int_{I_{n}}\left(k^{-1}\left(z^{n}-z^{n-1}, u^{n}\right)-a\left(z^{n-1}, u^{n}\right)\right) d t \\
& -\sum_{n=1}^{N} \int_{I_{n}}\left(k^{-1}\left(z^{n}-z^{n-1}, \tilde{u}^{n}\right)-a\left(z^{n-1}, \tilde{u}^{n}\right)\right) d t \\
= & -\sum_{n=1}^{N} \int_{I_{n}}\left(k^{-1}\left(z^{n-1}, u^{n}-u^{n-1}\right)+a\left(z^{n-1}, u^{n}\right)\right) d t \\
& +\sum_{n=1}^{N} \int_{I_{n}} k^{-1}\left[\left(z^{n}, u^{n}\right)-\left(z^{n-1}, u^{n-1}\right)\right] d t \\
& -\sum_{n=1}^{N} \int_{I_{n}}\left(k^{-1}\left(z^{n}-z^{n-1}, \tilde{u}^{n}\right)-a\left(z^{n-1}, \tilde{u}^{n}\right)\right) d t \\
= & -\left(z^{0}, u^{0}\right)-\sum_{n=1}^{N} \int_{I_{n}}\left(k^{-1}\left(z^{n}-z^{n-1}, \tilde{u}^{n}\right)-a\left(z^{n-1}, \tilde{u}^{n}\right)\right) d t,
\end{aligned}
$$

where the last line follows from (3) and $z^{N}=0$. Now from summation by parts we have

$$
\begin{aligned}
\|\eta\|_{0,0}^{2} & =-\left(z^{0}, u^{0}-\tilde{u}^{0}\right)+\sum_{n=1}^{N} \int_{I_{n}}\left(k^{-1}\left(z^{n-1}, \tilde{u}^{n}-\tilde{u}^{n-1}\right)+a\left(z^{n-1}, \tilde{u}^{n}\right)\right) d t \\
& =-\sum_{n=1}^{N} \int_{I_{n}} k^{-1}\left(\theta^{n}-\theta^{n-1}, z^{n-1}\right) d t .
\end{aligned}
$$

In the last step we subtracted the equation

$$
0=\sum_{n=1}^{N} \int_{I_{n}}\left(k^{-1}\left(y^{n}-y^{n-1}, \phi\right)+a(y, \phi)\right) d t
$$

and used the fact that

$$
\int_{I_{n}} a\left(y-\tilde{u}^{n}, \phi\right) d t=0
$$

for $\phi=z^{n-1}$. Also, the first term is zero since $\tilde{u}^{0}=u^{0}$. Summation by parts gives

$$
\|\eta\|_{0,0}^{2}=\sum_{n=1}^{N}\left(z^{n}-z^{n-1}, \theta^{n-1}\right),
$$

where we used the fact that $\left(\theta^{0}, z^{0}\right)=\left(\left(I-\mathscr{P}_{h}^{0}\right) v, z^{0}\right)=0$.

By setting $\phi=z^{n-1}-z^{n}$ in (83) and using summation by parts, it follows that

$$
\sum_{n=1}^{N} k^{-1}\left\|z^{n}-z^{n-1}\right\|_{0}^{2} \leq \sum_{n=1}^{N} k\left\|\eta^{n}\right\|_{0}^{2}=\|\eta\|_{0,0}^{2} .
$$


By the Cauchy-Schwarz inequality applied to (86) we have

$$
\begin{aligned}
\|\eta\|_{0,0}^{2} & \leq\left(\sum_{n=1}^{N} k\left\|\theta^{n-1}\right\|_{0}^{2}\right)^{1 / 2}\left(\sum_{n=1}^{N} k^{-1}\left\|z^{n}-z^{n-1}\right\|_{0}^{2}\right)^{1 / 2} \\
& \leq \sum_{n=1}^{N} k\left\|\theta^{n-1}\right\|_{0}^{2} .
\end{aligned}
$$

By (79) we have the required estimate for $\eta$, and the estimate (82) now follows from (78).

We now move on to the $L^{\infty}$ estimate.

Theorem 4. There exists a constant $C$ independent of $h, u$, and $y$ such that for any $0 \leq n \leq N$

$$
\left\|u^{n}-y^{n}\right\|_{0} \leq C\left(1+\ln \left(\frac{t_{n}}{k}\right)\right)\left(k+h^{2}\right)\|y\|_{L^{\infty}\left(0, T ; H^{2}(\Omega)\right)} .
$$

Proof. Let $z \in V_{h}^{0} \otimes V_{k}$ satisfy $z^{N}=\eta^{N}$ and

$$
k^{-1}\left(z^{n}-z^{n-1}, \phi\right)-a\left(z^{n-1}, \phi\right)=0, \quad \phi \in V_{h}^{0} .
$$

Letting $\phi=u^{n}-\tilde{u}^{n}$ and integrating in time, we obtain

$$
\sum_{n=1}^{N} \int_{I_{n}}\left[k^{-1}\left(z^{n}-z^{n-1}, u^{n}-\tilde{u}^{n}\right)-a\left(z^{n-1}, u^{n}-\tilde{u}^{n}\right)\right] d t=0 .
$$

Since $\tilde{u}^{0}=u^{0}$, summing by parts gives

$$
\left\|\eta^{N}\right\|_{0}^{2}=-\sum_{n=1}^{N} \int_{I_{n}}\left[k^{-1}\left(u^{n}-\tilde{u}^{n}-\left(u^{n-1}-\tilde{u}^{n-1}\right), z^{n-1}\right)+a\left(z^{n-1}, u^{n}-\tilde{u}^{n}\right)\right] d t .
$$

From (83) and (84) this becomes

$$
\left\|\eta^{N}\right\|_{0}^{2}=-\sum_{n=1}^{N} \int_{I_{n}}\left[k^{-1}\left(\theta^{n}-\theta^{n-1}, z^{n-1}\right)+a\left(\theta, z^{n-1}\right)\right] d t .
$$

Summing by parts and recalling that $\left(\theta^{0}, z^{0}\right)=\left(\left(I-\mathscr{P}_{h}^{0}\right) v, z^{0}\right)=0$, we find

$$
\left\|\eta^{N}\right\|_{0}^{2}=\left(\theta^{N}, z^{N}\right)-\sum_{n=1}^{N} \int_{I_{n}}\left[k^{-1}\left(\theta^{n-1}, z^{n}-z^{n-1}\right)+a\left(\theta, z^{n-1}\right)\right] d t .
$$

From (85) we obtain, since $z^{N}=\eta^{N}$,

$$
\begin{aligned}
\left\|\eta^{N}\right\|_{0}^{2} & =\left(\theta^{N}, z^{N}\right)-\sum_{n=1}^{N}\left(z^{n}-z^{n-1}, \theta^{n-1}\right) \\
& \leq\left\|\theta^{N}\right\|_{0}\left\|\eta^{N}\right\|_{0}+\max _{1 \leq n \leq N}\left\|\theta^{n}\right\|_{0} \sum_{n=1}^{N}\left\|z^{n}-z^{n-1}\right\|_{0} .
\end{aligned}
$$

From $[14$, p. 166] we have

$$
\sum_{n=1}^{N}\left\|z^{n}-z^{n-1}\right\|_{0} \leq C\left(1+\ln \left(\frac{T}{k}\right)\right)\left\|z^{N}\right\|_{0}=C\left(1+\ln \left(\frac{T}{k}\right)\right)\left\|\eta^{N}\right\|_{0},
$$


so

$$
\left\|\eta^{N}\right\|_{0} \leq C\left(1+\ln \left(\frac{T}{k}\right)\right) \max _{1 \leq n \leq N}\left\|\theta^{n}\right\|_{0} .
$$

From (80) and the fact that $N$ can be replaced by any $n$ the proof is complete.

\section{NUMERICAL RESULTS}

In this section we discuss a practical implementation of our method on some problems with both smooth and nonsmooth boundary data. We let $A=-\Delta$. Our scheme, which consists of piecewise linear finite elements in space and the implicit backward Euler method in time, is standard except for the handling of the boundary conditions. We use a preconditioned conjugate gradient method to solve the linear systems that arise on each time step. An incomplete Cholesky decomposition provides the preconditioner.

The numerical results demonstrate clearly the advantages of a robust method. In a smooth example the optimal $O\left(h^{2}\right)$ convergence rate is achieved. In experiments with boundary data that has jumps and discontinuities in time and space, we found the rate of convergence over the range tested is more like $O\left(h^{\gamma}\right)$, where $\gamma>1 / 2$. Previous methods that required the approximation to be zero on the boundary could achieve at most an $O\left(h^{1 / 2}\right)$ convergence rate.

Our practical evaluation of the boundary conditions requires some discussion. To find $u^{n+1}$ from $u^{n}$, we need the boundary function $Q g$ restricted to the interval in time $I_{n}$, which is given by

$$
\int_{I_{n}}\langle(I-Q) g, \chi\rangle d t=0, \quad \chi \in V_{h}(\Gamma) .
$$

Let $\left\{\phi_{1}, \ldots, \phi_{J}\right\}$ be a basis for $V_{h}(\Gamma)$. We have, on $I_{n}, Q g=\sum_{j=1}^{J} c_{j} \phi_{j}$, and taking $\chi=\phi_{i}$ gives

$$
\sum_{j=1}^{J} c_{j} k\left\langle\phi_{j}, \phi_{i}\right\rangle=\int_{I_{n}}\left\langle g, \phi_{i}\right\rangle d t .
$$

To simplify the left side, we approximate the inner product by the trapezoid rule; the matrix then becomes diagonal. On the right side of the equation we used the trapezoid rule in the smooth case and the rectangle rule with a large number of subdivisions in the rough data case. In all experiments we took $\Omega=(0,1) \times(0,1), T=0.1$, used a uniform mesh to discretize $\Omega$, and chose $k=C h^{2}$.

The results for our experiment with smooth boundary data are displayed in Table 1 (see next page). We took as the known solution

$$
y\left(x_{1}, x_{2}\right)=e^{-\pi^{2} t / 2} \sin \left(\frac{\pi x_{1}}{2}\right) \sin \left(\frac{\pi x_{2}}{2}\right) .
$$

The order of convergence was computed by the formula

$$
\text { Rate }=\ln \left(E_{2} / E_{1}\right) / \ln \left(h_{2} / h_{1}\right),
$$

where $E_{1}$ and $E_{2}$ are errors on successive meshes, and $h_{1}$ and $h_{2}$ are successive triangle diameters on these meshes. As predicted, we obtain an $O\left(h^{2}\right)$ rate of convergence. 
TABLE 1. Smooth $g$

\begin{tabular}{|c|c|c|c|}
\hline Mesh & $N$ & Error & Rate \\
\hline $4 \times 4$ & 2 & $0.456(-1)$ & - \\
$8 \times 8$ & 8 & $0.112(-1)$ & 2.03 \\
$16 \times 16$ & 32 & $0.280(-2)$ & 2.00 \\
$32 \times 32$ & 128 & $0.701(-3)$ & 2.00 \\
\hline
\end{tabular}

Our solution in the second experiment has a jump in time in its boundary data, $g=0$ for $t<\tilde{t}$ and $g=1$ for $t \geq \tilde{t}$, where $\tilde{t}=0.07071$ and $v \equiv 0$. The true solution to this problem is obtained by separation of variables. We find $y(\cdot, t)=1+z(\cdot, t-\tilde{t})$, where

$$
z\left(x_{1}, x_{2}, t\right)=-16 \sum_{n, m=1}^{\infty} \frac{1}{a_{n} b_{m}} e^{-\left(a_{n}^{2}+b_{m}^{2}\right) t} \sin \left(a_{n} x_{1}\right) \sin \left(b_{m} x_{2}\right),
$$

$a_{n}=(2 n-1) \pi, b_{m}=(2 m-1) \pi, z=0$ on $\Gamma$, and $z=-1$ at $t=0$ on $\Omega$. We evaluated all boundary integrals using the trapezoid rule, splitting the integral on the interval $I_{j}$ that contains $\tilde{t}$ into two pieces, one on each side of the jump in the boundary data. Table 2 has the results for this case. It is not surprising that the convergence is better than $h^{1 / 2}$, since the solution is smooth except near $\tilde{t}$.

TABLE 2. $g$ discontinuous in time

\begin{tabular}{|c|c|c|c|}
\hline Mesh & $N$ & Error & Rate \\
\hline $4 \times 4$ & 2 & 0.728 & - \\
$8 \times 8$ & 8 & $0.528(-1)$ & 3.79 \\
$16 \times 16$ & 32 & $0.150(-1)$ & 1.82 \\
$32 \times 32$ & 128 & $0.508(-2)$ & 1.56 \\
\hline
\end{tabular}

In the final test the boundary data is given by

$$
g\left(x_{1}, x_{2}, t\right)=\operatorname{sgn}\left(\sin \left(\frac{4 \pi t}{T}+\sqrt{3}\right) \sin \left(4 \pi x_{1}+\sqrt{2}\right) \sin \left(6 \pi x_{2}+e\right)\right),
$$

where sgn is the signum function. Here, $g$ has discontinuities in both space and time. Since we do not know the true solution in this case, we compared our approximations to a finite element approximation obtained on a $64 \times 64$ mesh with 512 timesteps. Table 3 has the results, which again show the convergence rate is better than $h^{1 / 2}$. 
TABLE 3. $g$ rough

\begin{tabular}{|c|c|c|c|}
\hline Mesh & $N$ & Error & Rate \\
\hline $4 \times 4$ & 2 & $0.118(+1)$ & - \\
$8 \times 8$ & 8 & 0.671 & .814 \\
$16 \times 16$ & 32 & 0.274 & 1.29 \\
$32 \times 32$ & 128 & 0.132 & 1.05 \\
\hline
\end{tabular}

\section{BIBLIOGRAPHY}

1. I. Babuška and J. Osborn, Eigenvalue problems, Handbook of Numerical Analysis, vol. II (P. G. Ciarlet and J. L. Lions, eds.), North-Holland, Amsterdam, 1991, pp. 641-787.

2. R. Bank and T. Dupont, An optimal order process for solving finite element equations, Math. Comp. 36 (1981), 35-51.

3. J. H. Bramble, J. Pasciak, and A. H. Schatz, The construction of preconditioners for elliptic problems by substructuring. I, Math. Comp. 47 (1986), 103-134.

4. J. H. Bramble and V. Thomée, Discrete time Galerkin methods for a parabolic boundary value problem, Ann. Mat. Pura Appl. 101 (1974), 115-152.

5. P. L. Butzer and H. Berens, Semi-groups of operators and approximation, Springer-Verlag, Berlin, 1967.

6. G. Choudury, Fully discrete Galerkin approximations of parabolic boundary-value problems with nonsmooth boundary data, Numer. Math. 57 (1990), 179-203.

7. P. G. Ciarlet, The finite element method for elliptic problems, North-Holland, Amsterdam, 1978.

8. K. Eriksson and C. Johnson, Error estimates and automatic time step control for nonlinear parabolic problems. I, SIAM J. Numer. Anal. 24 (1987), 12-23.

9. K. Eriksson, C. Johnson, and V. Thomée, Time discretization of parabolic problems by the discontinuous Galerkin method, RAIRO Modél. Math. Anal. Numér. 19 (1985), 611-643.

10. G. J. Fix, M. D. Gunzburger, and J. S. Peterson, On finite element approximations of problems having inhomogeneous essential boundary conditions, Comput. Math. Appl. 9 (1983), 687-700.

11. D. French and J. T. King, Approximation of an elliptic control problem by the finite element method, Numer. Funct. Anal. Optim. 12 (1991), 299-314.

12. P. Grisvard, Behavior of the solutions of an elliptic boundary value problem in a polygonal or polyhedral domain, Numerical Solution of Partial Differential Equations. III (B. Hubbard, ed.), Academic Press, New York, 1976, 207-274.

13. P. Grisvard, Elliptic problems in nonsmooth domains, Pitman, Boston, 1985.

14. C. Johnson, Numerical solutions of partial differential equations by the finite element method, Cambridge Univ. Press, Cambridge, 1987.

15. C. Johnson, Y.-Y. Nie, and V. Thomée, An a posteriori error estimate and adaptive timestep control for a backward Euler discretization of a parabolic problem, SIAM J. Numer. Anal. 27 (1990), 277-291.

16. G. Knowles, Finite element approximation of parabolic time optimal control problems, SIAM J. Control. Optim. 20 (1982), 414-427.

17. I. Lasiecka, Convergence estimates for semidiscrete approximations of nonselfadjoint parabolic equations, SIAM J. Numer. Anal. 21 (1984), 894-909.

18. __ Galerkin approximations of abstract parabolic boundary value problems with rough boundary data $-L_{p}$ theory, Math. Comp. 47 (1986), 55-75. 
19. __ Unified theory for abstract parabolic boundary problems-A semigroup approach, Appl. Math. Optim. 6 (1980), 287-333.

20. J. L. Lions and E. Magenes, Nonhomogeneous boundary value problems and applications. 1, 2, Springer-Verlag, Berlin, 1972.

21. V. Thomée, Galerkin finite element methods for parabolic problems, Lecture Notes in Math., vol. 1054, Springer-Verlag, Berlin, 1984.

22. R. Winther, Error estimates for a Galerkin approximation for a parabolic control problem, Ann. Mat. Pura Appl. 104 (1978), 173-206.

Department of Mathematical Sciences, University of Cincinnati, Cincinnati, Ohio 45221-0025

E-mail address: french@ucunix.san.uc.edu

E-mail address: kingjt@ucbeh.san.uc.edu 